\title{
COMPARAÇÃO ENTRE DUAS TÉCNICAS DE COLORAÇÃO DE ENCÉFALOS HUMANOS
}

\section{COMPARATIVE ANALYSIS OF HUMAN BRAIN WITH TWO DIFFERENT STAINING TECHINIQUES}

\author{
Thiago Teza MERINI ${ }^{1}$, Lucas Kraeski KRUM ${ }^{1}$, Joelmir COLMAN1, Renato Van Wilpe \\ BACH $^{2}$, Marcelo Rezende Young BLOOD ${ }^{3}$, Carlos Henrique Ferreira CAMARGO ${ }^{4}$ \\ ${ }^{1}$ Acadêmicos do Curso de Medicina. Universidade Estadual de Ponta Grossa (UEPG). \\ Avenida Carlos Cavalcanti, 4748, Uvaranas, 84030-900, Ponta Grossa, PR, Brasil. \\ ${ }^{2}$ Professor Assistente de Anatomia Humana e de Pediatria Clínica e Cirúrgica Departamento \\ de Medicina. Universidade Estadual de Ponta (UEPG). Avenida Carlos Cavalcanti, 4748, \\ Uvaranas, 84030-900, Ponta Grossa, PR, Brasil. \\ ${ }^{3}$ Professor Colaborador de Neurologia. Departamento de Medicina. Universidade Estadual \\ de Ponta (UEPG). Avenida Carlos Cavalcanti, 4748, Uvaranas, 84030-900, Ponta Grossa, \\ PR, Brasil. \\ ${ }^{4}$ Autor para contato: Professor Adjunto de Neurologia e de Fundamentos de Neurociência. \\ Departamento de Medicina. Universidade Estadual de Ponta (UEPG). Avenida Carlos \\ Cavalcanti, 4748, Uvaranas, 84030-900, Ponta Grossa, PR, Brasil. E-mail: chcamargo@ \\ uol.com.br
}

Data de recebimento: $18 / 11 / 2013$

Data da aprovação: 21/01/2014

\section{RESUMO}

O estudo da neuroanatomia de encéfalos humanos em cortes pode ser facilitado utilizando-se métodos de coloração para a substância cinzenta. O objetivo do estudo foi determinar, entre as técnicas de Mainland e de Barnard, Robert e Brown, qual teria melhores resultados para aplicação em laboratórios de anatomia. Foram testadas as técnicas em trinta cortes coronais de hemisfério cerebral, comparando entre si e analisando objetivamente de acordo com três parâmetros: grau de diferenciação entre as substâncias branca e cinzenta do córtex; presença de linha única e contínua separando a substância branca do córtex cerebral; grau de impregnação da coloração na substância branca. Depois de finalizada a coloração das peças, foi realizada uma análise objetiva da melhor visualização macroscópica da substância cinzenta. Foram atribuídas pontuações de 0 a 3 conforme a presença destes parâmetros, cada corte recebendo pontuação total que variava de 0 a 9. Após análise estatística, a técnica de Mainland apresentou resultado 6,13 $\pm 1,64$ e a de Barnard, Robert e Brown de 3,33 $\pm 2,47(\mathrm{p}<0,001)$. Além desse resultado, em relação ao tempo dispendido para cada técnica e a facilidade do preparo de cada uma, o resultado a que chegamos concluiu que a técnica de Mainland apresentou-se como melhor opção quando comparada com a técnica de Barnard, Robert e Brown.

Palavras-chave: Neuroanatomia. Técnicas de coloração. Encéfalo humano

\begin{abstract}
The neuroanatomy study of human brain can be facilitated by use of methods for staining of gray matter.The aim of this study was to determine, between staining techniques of Mainland and Barnard, Robert and Brown, which would be best for use in anatomy laboratories. The techniques were applied in coronal cerebral hemisphere, comparing
\end{abstract}


each other and analyzing according to three parameters: degree of differentiation between white and gray matter of the cortex, presence of a single continuous line separating the white matter of the cerebral cortex; degree of impregnation of the staining in the white matter. An objective analysis of the best macroscopic visualization of gray matter was performed. Scores were assigned from 0 to 3 according to the presence of these parameters, with receiving total score ranging from 0 to 9 . After statistical analysis, the Mainland technique presented result $6.13 \pm 1.64$ and Barnard, Robert and Brown, $3.33 \pm$ $2.47(\mathrm{p}<0.001)$. In relation to the time spent for each technique, and facility to prepare, the result which we concluded that the Mainland technique presented as better option than the Barnard, and Robert and Brown technique.

Keywords: Neuroanatomy. Staining techniques. Human brain.

\section{Introdução}

O estudo da neuroanatomia pode ser considerado muitas vezes de difícil entendimento pelos estudantes e de difícil demonstração por parte dos professores. (MENESES et al., 2004). Para auxiliar e complementar o estudo teórico é essencial a neuroanatomia prática, visando levar à compreensão e identificação dos componentes do sistema nervoso. Porém, a diferenciação entre substâncias branca e cinzenta pode ser difícil a olho nu e a maioria das estruturas encefálicas não são facilmente identificadas nas peças anatômicas convencionais. (ARRUDA, 1999). Tendo isto em vista, não é recente a busca por métodos que auxiliem técnicos, estudantes e professores no ensino e aprendizagem das neurociências. (RODRIGUES, 2010). Uma abordagem interessante é a da coloração da substância cinzenta para diferenciá-la da substância branca, pois é mais fácil e rápida do que a coloração da substância branca. Esse tipo de técnica auxilia na identificação do córtex cerebral e dos núcleos da base. (MENESES et al., 2004).

Mainland (1926) publicou um artigo sobre uma técnica de coloração, propondo um método simples e relativamente rápido para corar de azul a substância cinzenta. O tempo total do procedimento é de aproximadamente 24 horas e todas as soluções são utilizadas em temperatura ambiente. Mais de vinte anos depois foi proposta a técnica de Barnard, Robert e Brown (1949), que utiliza solução de Mulligan (definida nos métodos) para corar, também de azul, a substância cinzenta. Esta técnica é mais rápida do que a primeira, mas é mais trabalhosa devido à preparação desta solução e a utilização dela a $60^{\circ} \mathrm{C}$.

Apesar de todos os avanços na metodologia de ensino das neurociências, os métodos de coloração ainda têm seu lugar no ensino, devido à simplicidade de execução e os bons resultados obtidos. (RODRIGUES, 2010). Não foram encontrados dados na literatura científica que comparassem a eficácia das duas técnicas. Este foi, portanto, o objetivo do presente estudo.

\section{Materiais e método}

Após aprovação pelo Comitê de Ética em Pesquisa de Seres Humanos, da Universidade Estadual de Ponta Grossa (COEP-UEPG), dois encéfalos humanos, pertencentes ao Laboratório de Anatomia Humana da UEPG, foram obtidos para realização do estudo. As peças estavam colocadas em solução fixadora de formol a $10 \%$. Foram retiradas e desprezadas todas as meninges e os vasos da superfície do encéfalo.

\section{Corte e separação das peças anatômicas}

As peças anatômicas foram submetidas à secção através de cortes coronais seriados de espessura de $10 \mathrm{~mm}$, apoiando-se o material sobre um superfície plana, com auxílio de marcadores com $10 \mathrm{~mm} \mathrm{e}$ lâmina com corte afiado.

Foram separados 30 cortes de hemisfério cerebral, 15 de cada cérebro, para as técnicas de Mainland e de Barnard, Robert \& Brown, respectivamente.

\section{Técnicas de coloração}

Como preparo, os cortes foram colocados em água oxigenada a 10 volumes por 24 horas para clareamento e então lavagem em água corrente. Prosseguiu-se com a coloração das peças de acordo com as técnicas de coloração. 
A técnica de Mainland (1926) foi realizada da seguinte forma: os cortes foram submersos por $5 \mathrm{mi}$ nutos em solução de cloreto férrico a $1 \%$, então foram lavados em água corrente. Após essa etapa, os cortes foram submersos em uma solução de ferrocianeto de potássio a $1 \%$ até obter a coloração desejada (normalmente por cerca de 30 segundos), e lavada novamente em água corrente. Depois de coloridas, as peças foram colocadas em solução de ácido nítrico a $1 \%$ por 24 horas. Para conservação, as peças permaneceram em solução de formol a $5 \%$.

Para a técnica de Barnard, Robert \& Brown (1949), utilizou-se a solução de Mulligan, que é composta por cristais de fenol $4 \mathrm{~g}$ ou solução de fenol $89 \%$, sulfato de cobre $0,5 \mathrm{~g}$, ácido clorídrico $1,25 \mathrm{ml}$, água destilada $100 \mathrm{ml}$. Os cortes então foram colocados em solução de Mulligan aquecida a 60 graus por 2 ou 3 minutos, lavou-se em água morna durante 1 minuto, então os cortes foram emergidos na solução de cloreto férrico a $1 \%$ por 1 minuto e lavados em água corrente durante 1 minuto. No passo seguinte, as peças foram colocadas em solução de ferrocianeto de potássio a $1 \%$ por 1 minuto, em seguida retiradas e lavadas em água morna corrente por 1 minuto. Para conservação, foi utilizada solução de formol a 10\% com ácido clorídrico, fazendo uma solução a $2 \%$.

\section{Análise comparativa das peças coloridas}

Após finalizadas as peças, foi realizada uma análise objetiva, utilizando-se três critérios: (1) grau de diferenciação entre as substâncias branca e cinzenta; (2) presença de linha única e contínua separando a substância branca do córtex que a envolve; e (3) grau de impregnação da coloração em outros locais da substância branca. Estes parâmetros foram quantificados a partir dos critérios de Meneses et al. (2004). Estipulou-se uma pontuação de 0 a 3 para cada corte de hemisfério.

A nota 0 foi atribuída aos cortes de hemisfério que apresentaram melhor distinção entre substância branca e cinzenta numa área menor que $25 \%$ da superfície total do corte; a nota 1 indicou que essa melhor diferenciação foi possível numa área de $25 \%$ a $50 \%$ da superfície de corte; a nota 2 mostrou a maior nitidez da distinção numa área de $50 \%$ a $75 \%$; e a nota 3 atribuída à superfície maior que $75 \%$.

$\mathrm{O}$ parâmetro 2 referiu-se à presença de linha única e contínua separando a substância branca do córtex cerebral que a envolvia. Para análise deste parâmetro atribuiu-se pontuação de 0 a 3 para cada corte de hemisfério. A nota 0 indicou presença dessa linha única e contínua em área menor que $25 \%$ da superfície total do corte; a nota 1 mostrou presença da linha em área de $25 \%$ a $50 \%$ da superfície total do corte; a nota 2 , linha em uma área de $50 \%$ a $75 \%$ da superfície total de corte; e a nota 3 , em área maior que $75 \%$.

O parâmetro 3 indicou o grau de impregnação da coloração em outros locais de substância branca, como a coroa radiada, a cápsula interna e a substância branca do lobo temporal. Avaliou-se a extensão e a tonalidade com que essa impregnação foi observada. Do mesmo modo, atribuiu-se pontuação de 0 a 3 para avaliar este critério. A nota 0 indicou substância branca impregnada pelo corante em área superior a 75\% da superfície total do corte; a nota 1 referiu-se à presença de substância branca impregnada pelo corante em área de 50\% a 75\% de superfície total do corte; a nota 2 , impregnação pelo corante em área de $25 \%$ a $50 \%$ da superfície total; e a nota 3 , em área menor que $25 \%$ da superfície total de corte.

\section{Análise estatística}

As diferenças estatísticas das médias entre os grupos foram determinadas utilizando-se o teste $t d e$ Student unicaudal para distribuições normais, e o teste de Mann-Whitney para distribuições não normais. Os resultados estão apresentados como média \pm DP (desvio padrão). Toda a análise estatística foi realizada com os programas Microsoft Office Excel for Windows versão 2011 e Statistica for Windows versão 99. As diferenças foram consideradas significativas quando $\mathrm{p}<0.05$.

\section{Resultados}

$\mathrm{Na}$ avaliação dos cortes realizados, a coloração de Mainland apresentou resultado somado total de $6,13 \pm 1,64$, contra 3,33 $\pm 2,47$ para a coloração de Barnard, Robert e Brown ( $<<0,001)$. Quando analisado isoladamente cada parâmetro, obtivemos os seguintes resultados: diferenciação entre substân-

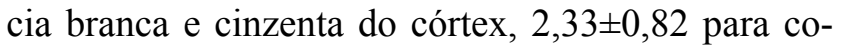

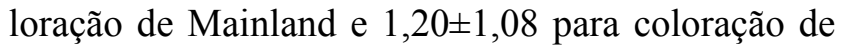
Barnard, Robert e Brown ( $\mathrm{p}=0,0015)$; presença de linha única e contínua separando a substância branca do córtex, 2,13 $\pm 0,74$, para coloração de Mainland e $1,40 \pm 0,83$ para coloração de Barnard, Robert e Brown 


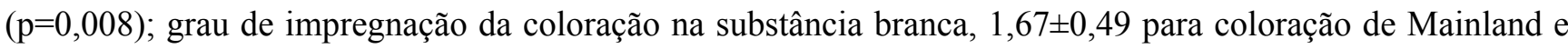
média $0,73 \pm 0,80$ para coloração de Barnard, Robert e Brown $(\mathrm{p}<0,001)$ (Figura 1).

Figura 1 - Comparação entre as técnicas de Mainland e de Barnard, Robert e Brown. Nos três critérios isoladamente e no total os resultados foram diferentes de forma estatisticamente significante $(\mathrm{p}<0,001)$

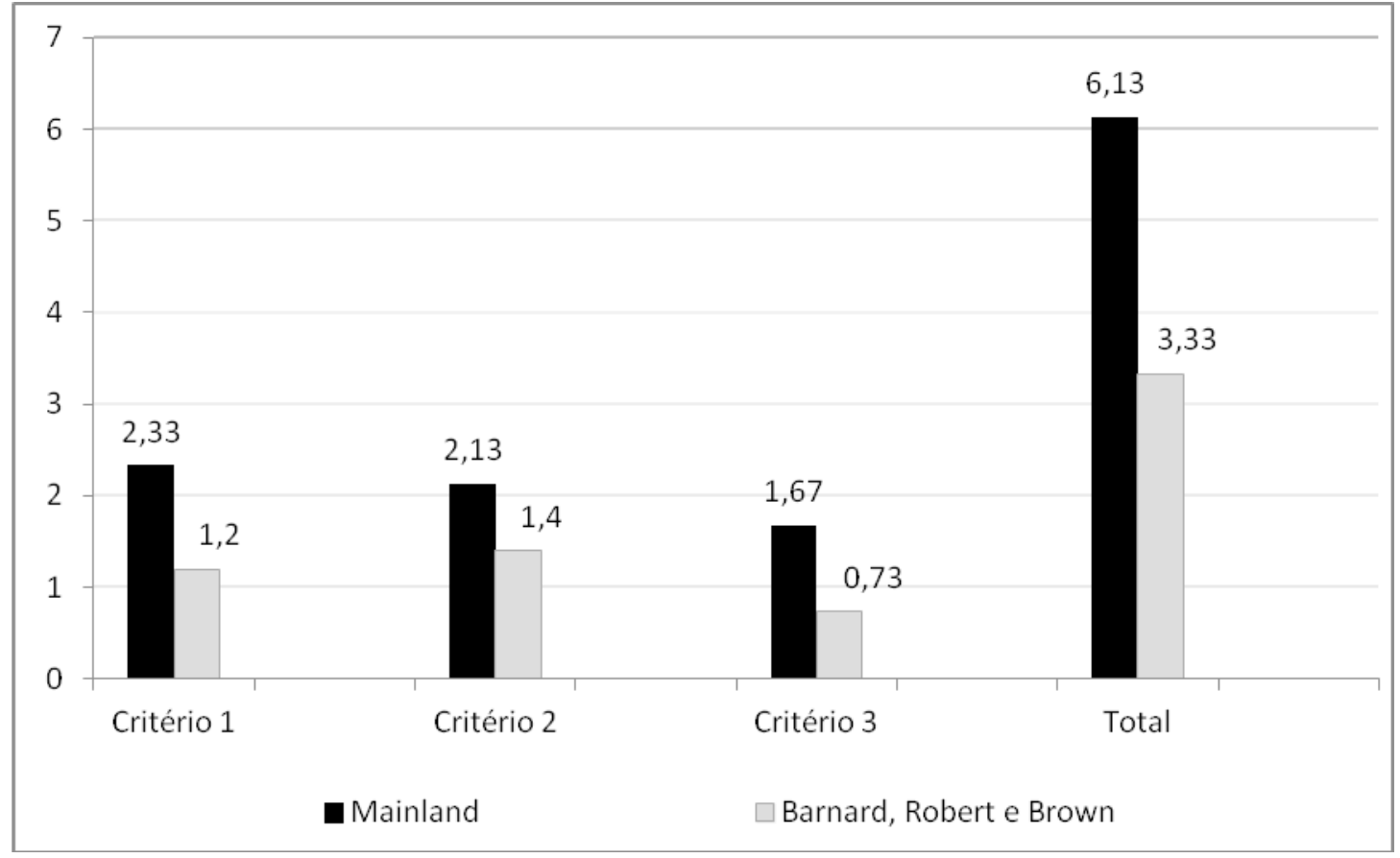

A Figura 2 exemplifica um corte encefálico coronal submetido à técnica de Mainland, enquanto a Figura 3 monstra um corte axial corado pela técnica de Barnard, Robert e Brown.

Figura 2 - Corte encefálico coronal submetido à técnica de Mainland

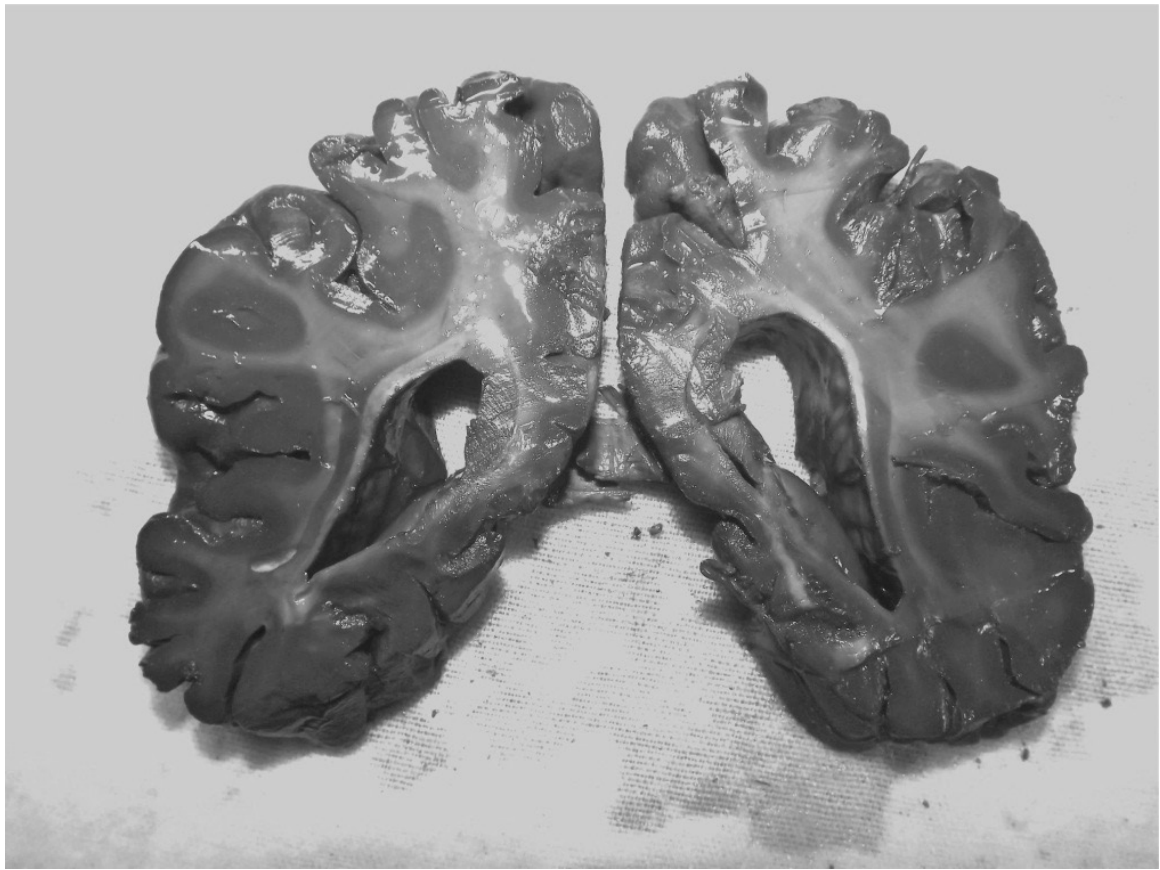


Figura 3- Corte cerebral corado pela técnica de Barnard, Robert e Brown

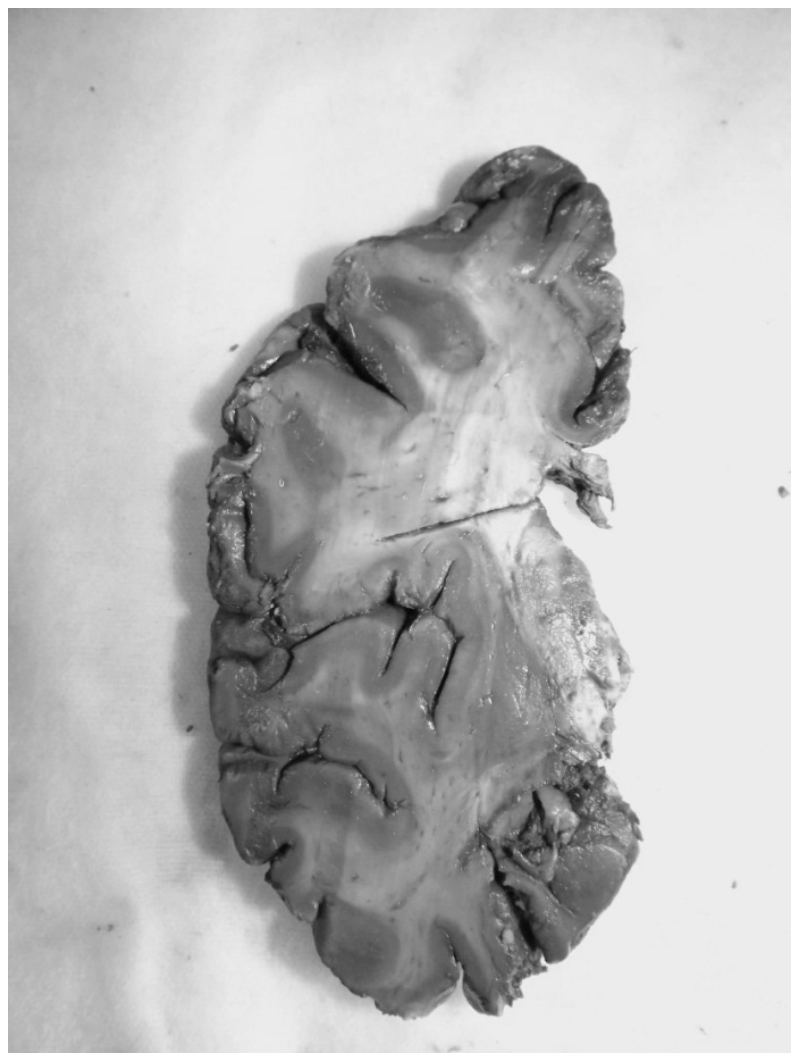

\section{Discussão}

A grande revolução tecnológica dos métodos de neuroimagem na medicina, como o uso da tomografia computadorizada e da ressonância magnética, tem demonstrado detalhes anatômicos precisos. Junto a esses avanços, há uma necessidade cada vez maior do estudo anatômico prévio das estruturas encefálicas para melhor compreensão das estruturas de substância cinzenta e branca, justificando a pesquisa de métodos que favoreçam o estudo da neuroanatomia. (BLACKSTAD, 1993).

O presente estudo demonstrou que as técnicas de coloração empregadas foram úteis para uma melhor visualização da delimitação entre a substância cinzenta e a substância branca, uma das principais vantagens do emprego dos métodos de coloração. (RODRIGUES, 2010). O método de Mainland foi superior ao método de Barnard, Robert e Brown. Meneses et al. (2004), em um trabalho de comparação entre as técnicas de Green, Mulligan e Barnard, Robert e Brown, obtiveram melhores resultados desta última, sendo que apresentou melhor grau de diferen- ciação entre as substâncias branca e cinzenta. Entretanto, esses autores não usaram a técnica de Mainland que, tanto em parâmetros subjetivos quanto objetivos, foi superior à técnica de Barnard, Robert e Brown no presente estudo.

A superioridade da técnica de Mainland foi observada principalmente quando analisado o primeiro parâmetro comparativo, o qual verifica de modo objetivo a principal vantagem dos métodos de coloração: o grau de diferenciação entre as substâncias branca e cinzenta. Entre as desvantagens, principalmente analisada pelo terceiro parâmetro comparativo, esteve o grau de impregnação da coloração da substância branca em graus variáveis, algumas vezes bastante intensas. Essa característica foi marcante visualmente e estatisticamente superior pela técnica de Barnard, Robert e Brown.

$\mathrm{Na}$ tentativa de explicação dessas diferenças, podem-se evocar hipóteses de diferentes ações microscópicas celulares das duas técnicas nos tecidos formalizados. Outras técnicas consagradas, como a coloração baseada na impregnação do complexo de Golgi neuronal, servem como respaldo para a hipótese de que alguns fatores moleculares e celulares possam interferir nos resultados de melhor coloração. (FAIREN, 2005).

Dall'Oglio et al. (2010) propuseram que o uso de impregnação metálica de neurônios e células gliais, assim como as técnicas deste estudo, podem sofrer influências do metal utilizado. Alguns metais, como o dicromato de potássio, tetróxido de ósmio e nitrato de prata, podem ter uma reação de melhor ou pior eficácia, conforme a mistura e o tempo em que a peça seja exposta à substância química, podendo em muitos casos marcar a substância branca. A impregnação pela prata, por exemplo, aparentemente ocorre ao acaso, o que leva a um número de neurônios impregnados sem um padrão homogêneo de coloração celular. Nas técnicas comparadas neste estudo foram utilizados metais como o ferrocianeto de potássio, o sulfato de cobre e o cloreto férrico. Esses diferentes metais, com propriedades físico-químicas diferentes e provavelmente interação com estruturas celulares de modo desigual, podem explicar a impregnação da substância branca e a coloração irregular dos corpos neuronais da substância cinzenta de forma diferente com colorações diferentes. Outro aspecto que deve ser levado em consideração é que uma peça embebida 
em solução com formol 10\% com um pH abaixo de 2,5 pode produzir vários aspectos da impregnação irregular das células neuronais e gliais. (DALL'OGLIO et al. 2010).

Além de resultados melhores, a técnica de Mainland tem uma metodologia mais simples e mais rápida. A técnica de Barnard, Robert e Brown foi a que apresentou maior dificuldade na preparação, uma vez que necessita de quatro soluções diferentes, sendo uma delas aquecida a $60{ }^{\circ} \mathrm{C}$. Por outro lado, a técnica de Mainland exige, para sua realização, apenas três soluções, que não necessitam de aquecimento.

Concluímos que, quando comparadas as técnicas do trabalho, em relação à diferenciação de substância cinzenta e branca, obtivemos resultados que apontam a técnica de Mainland com melhor avaliação objetiva quando comparada com a técnica de Barnard, Robert e Brown. Portanto, sugerimos que os laboratórios de anatomia que desejem empregar técnicas de coloração utilizem o método de coloração de Mainland como primeira escolha.

\section{Agradecimentos}

Agradecemos ao Prof. Dr. Everson Krum e à Prof. Dra. Célia Regina Cerubelli pela ajuda com os reagentes químicos necessários para o preparo das colorações.

\section{Referências}

ARRUDA, W. O. O neurônio e o tecido nervoso. In Meneses MS. Neuroanatomia aplicada. Rio de Janeiro: Guanabara-Koogan, 1999: 2-27.

BARNARD, J. W; ROBERT, J. O.; BROWN, J. C. A simple macroscopic staining, and mouting, procedure for wet section from cadaver brains. Anat Rec.1949; 105:1-17.

BLACKSTAD, T. W. Computer methods in neuroanatomy: determining mutual orientation of whole neuronal arbors. Comput Biol Med 1993; 23:227-250.

DALL'OGLIO et al. The "single-section" Golgi method adapted for formalin-fixed human brain and light microscopy. Journal of Neuroscience Methods 2010; 189 $51-55$.

FAIREN, A. Pioneering a golden age of cerebral microcircuits: the births of the combined

Golgi-electron microscope methods. Neuroscience 2005; 136:607-14.

MAINLAND, D. Uber makroskopiche faerburg von Gehirnprraeparaten mit Berlinblau. Anat Anz. 1926; 65:85-88.
MENESES, M.S.; MONTANO PEDROSO J. C.; FUZZA R. F.; MILANO J. B. Análise comparativa de cortes de encéfalos humanos com coloração por três técnicas diferentes. Arq. Neuro-Psiquiatr. 2004; 62(2a): 276-281.

RODRIGUES, H. Técnicas anatômicas. 4. ed. Vitória: GM Gráfica e Editora, 2010. [S. 1.]. cap. 5. P. 173-201.

TEIVE HAG. Núcleos da base, estruturas correlatas e vias extrapiramidais. In Meneses MS. Neuroanatomia Aplicada. Rio de Janeiro: Guanabara-Koogan, 1999; 224236. 